\title{
Unusually Large, Unruptured Tubal Ectopic Pregnancy Mass in a Woman With Bilateral Tubal Obstruction Treated by Laparoscopy: A Case Report
}

\author{
Myounghwan Kim
}

\begin{abstract}
A tubal ectopic pregnancy mass usually measures $1.5-3.5 \mathrm{~cm}$, and ruptures if it grows beyond this size. The author encountered a case of an unruptured, tubal ectopic pregnancy mass measuring up to 7.3 $\mathrm{cm}$, containing an embryo with a crown-rump length (CRL) of 2.02 $\mathrm{cm}$, corresponding to 8 weeks, 4 days. To the author's knowledge, this is the largest reported singleton tubal ectopic pregnancy mass. A 36-year-old gravida 0 , para 0 woman presented with a history of mild vaginal bleeding, lower abdominal pain, and 8 weeks of amenorrhea. Vaginal bleeding started 3 weeks before, but she mistook this for menstruation. Her menstrual cycle ranged between 28 and 30 days and lasted 6 days. Her last menstrual period was about 8 weeks prior. She had an appendectomy 22 years prior. She was diagnosed with infertility due to tubal obstruction and underwent laparoscopic fimbrioplasty at another general hospital 13 years prior. She was told that her Fallopian tubes were still obstructed on hysterosalpingography after fimbrioplasty. She had not used contraception. On arrival in the emergency room, her serum beta-human chorionic gonadotropin (hCG) level was $15,944 \mathrm{mIU} / \mathrm{mL}$. The transvaginal ultrasound scan (TVUS) demonstrated an ectopic pregnancy mass measuring $7.3 \times 4.0 \mathrm{~cm}$ in the left adnexa. An embryo with CRL of $2.02 \mathrm{~cm}$ corresponding to 8 weeks, 4 days was noted in the ectopic pregnancy mass. Upon entering the pelvic cavity, a dark reddish-colored unruptured tubal ectopic pregnancy mass was noted. Laparoscopic salpingectomy was carried out. The right Fallopian tube was adherent to the right pelvic wall. Even though the Fallopian tubes are obstructed, pregnancy is still possible. Laboratory studies should always include a pregnancy test. A large tubal ectopic pregnancy mass could have developed when a blastocyst was implanted in a fused Fallopian tube that was adherent to the pelvic peritoneum and uterus.
\end{abstract}

Keywords: Ectopic pregnancy; Infertility; Tubal pregnancy; Laparoscopy; Tubal obstruction

Manuscript accepted for publication August 12, 2016

Department of Obstetrics and Gynecology, Sanggye Paik Hospital, Inje University, School of Medicine, Seoul, Korea. Email: myankim@paik.ac.kr

doi: http://dx.doi.org/10.14740/jmc2613w

\section{Introduction}

Ectopic pregnancy occurs when the developing blastocyst implants at a site other than the endometrium of the uterine cavity. Nearly $95 \%$ of ectopic pregnancies are implanted in the various segments of the Fallopian tubes. Of these, most are tubal ampullary implantations and $2-3 \%$ are interstitial (cornual) implantations. The remaining 5\% implant in the ovary, peritoneal cavity, cesarean section scar, or within the cervix. A cornual pregnancy mass can grow to a large size due to surrounding elastic myometrium, and may be discovered at an advanced gestational age [1].

However, a tubal ectopic pregnancy mass size is usually $1.5-3.5 \mathrm{~cm}[2,3]$, and ruptures if it grows beyond this size. The author experienced an unruptured huge tubal ectopic pregnancy mass measuring up to $7.3 \mathrm{~cm}$, containing an embryo with crown-rump length $(\mathrm{CRL})$ of $2.02 \mathrm{~cm}$, corresponding to 8 weeks, 4 days. To the author's knowledge, this is the largest reported, unruptured, tubal singleton ectopic pregnancy without a hematoma, measuring up to $7.3 \mathrm{~cm}$.

\section{Case Report}

A 36-year-old gravida 0 , para 0 woman presented with a history of mild vaginal bleeding, lower abdominal pain, and 8 weeks of amenorrhea. Vaginal bleeding started 3 weeks prior, but she mistook this for menstruation. Her menstrual cycle ranged between 28 and 30 days and lasted 6 days. Her last menstrual period was about 8 weeks prior. She had an appendectomy 22 years prior. She was diagnosed with infertility due to tubal obstruction and underwent laparoscopic fimbrioplasty at another general hospital 13 years prior. She was told that her Fallopian tubes were still obstructed on hysterosalpingography after fimbrioplasty. She had not used contraception. On arrival in the emergency room, vital signs showed blood pressure $120 / 70 \mathrm{~mm} \mathrm{Hg}$, pulse rate 82 beats per minute, and body temperature $36.5^{\circ} \mathrm{C}$. The serum beta-human chorionic gonadotropin (hCG) level was $15,944 \mathrm{mIU} / \mathrm{mL}$. The transvaginal ultrasound scan (TVUS) demonstrated an ectopic mass measuring $7.3 \times 4.0 \mathrm{~cm}$ in the left adnexa. An embryo with CRL of $2.02 \mathrm{~cm}$ corresponding to 8 weeks, 4 days of gestation was noted in the ectopic mass (Fig. 1). It was highly unlikely that medical treatment would be effective, and laparoscopic exploration was performed. Upon entering the pelvic cavity, a dark 


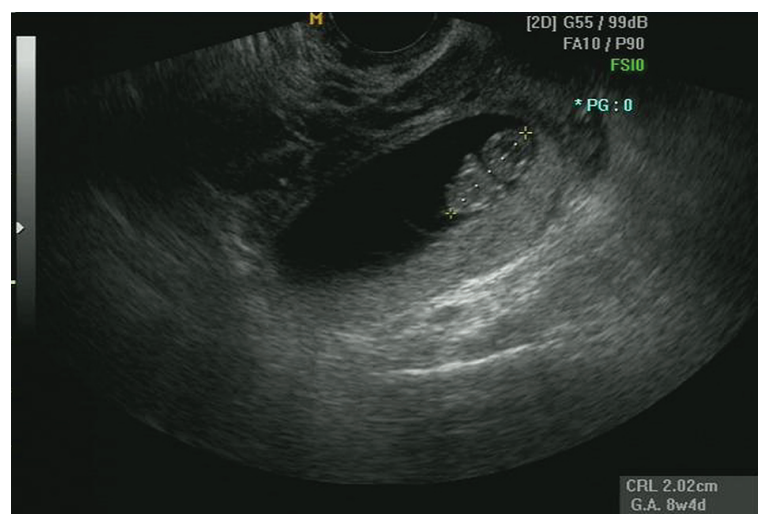

Figure 1. The transvaginal ultrasound scan (TVUS) demonstrated an ectopic mass measuring $7.3 \times 4.0 \mathrm{~cm}$ in the left adnexa, containing embryo with crown-rump length (CRL) of $2.02 \mathrm{~cm}$, corresponding to 8 weeks, 4 days of gestation.

bluish-colored, unruptured tubal mass was noted (Fig. 2a, b). During cutting of adhesions between the ectopic mass and surrounding structures (e.g., uterus, pelvic peritoneum), the mass ruptured and the embryo was expelled into the abdominal cavity (Fig. 2c). Laparoscopic left salpingectomy was carried out (Fig. 2d). The right Fallopian tube was also adherent to the right pelvic wall. The patient recovered successfully and was discharged on the second postoperative day. Follow-up in the Outpatient Department was satisfactory.

\section{Discussion}

The prevalence of ectopic pregnancy among women who visit an emergency department with first trimester bleeding, pain, or both ranges from $6 \%$ to $16 \%$ [4]. The overall incidence of ectopic pregnancy increased during the mid-20th century, pla- teauing at approximately 20 per 1,000 pregnancies in the early 1990s; the last time national data were reported by the Centers for Disease Control [5]. This rising incidence is strongly associated with an increased incidence of pelvic inflammatory disease [6]. The ectopic pregnancy maternal mortality rate declined because of early detection by high-resolution transvaginal ultrasonography and sensitive serum hCG concentration [7]. In this case, ectopic pregnancy was obvious, considering the serum hCG was $15,944 \mathrm{mIU} / \mathrm{mL}$ and an embryo with active fetal heartbeat was detected on TVUS. However, we still encounter "pregnancy unknown location" (PUL). In cases in which neither intrauterine nor extrauterine pregnancy is identified, we approach these with the concept of the hCG discriminatory zone (serum hCG level above which a gestational sac should be visualized by TVUS if an intrauterine pregnancy (IUP) is present). The hCG level of the discriminatory zone varies, but in most institutions, it is $1,500-2,000 \mathrm{mIU} / \mathrm{mL}[8$, 9]. Among women with a normal IUP, hCG levels vary widely, with almost $5 \%$ of women noted to have levels greater than $2,000 \mathrm{mIU} / \mathrm{mL}$. The highest level of $\mathrm{hCG}$ observed in a woman with a normal IUP was over $6,500 \mathrm{mIU} / \mathrm{mL}$ [10]. Doubilet and Benson concluded that the hCG discriminatory level should not be used to determine the management of a hemodynamically stable patient with suspected ectopic pregnancy, if sonography demonstrates no findings of intrauterine or ectopic pregnancy [10]. When women with early pregnancies of unknown implantation site are identified, ectopic precautions should be reviewed, and arrangements were made for followup hCG assessment. If a yolk sac, embryo, or fetus is identified within the uterus or the adnexa, then a diagnosis can be made. Importantly, not all adnexal masses represent an ectopic pregnancy, and integration of sonographic findings with other clinical information is necessary.

A cornual pregnancy mass can grow to a great size due to surrounding elastic myometrium, and may be discovered at an advanced gestational age [1]. However, tubal ectopic pregnan-
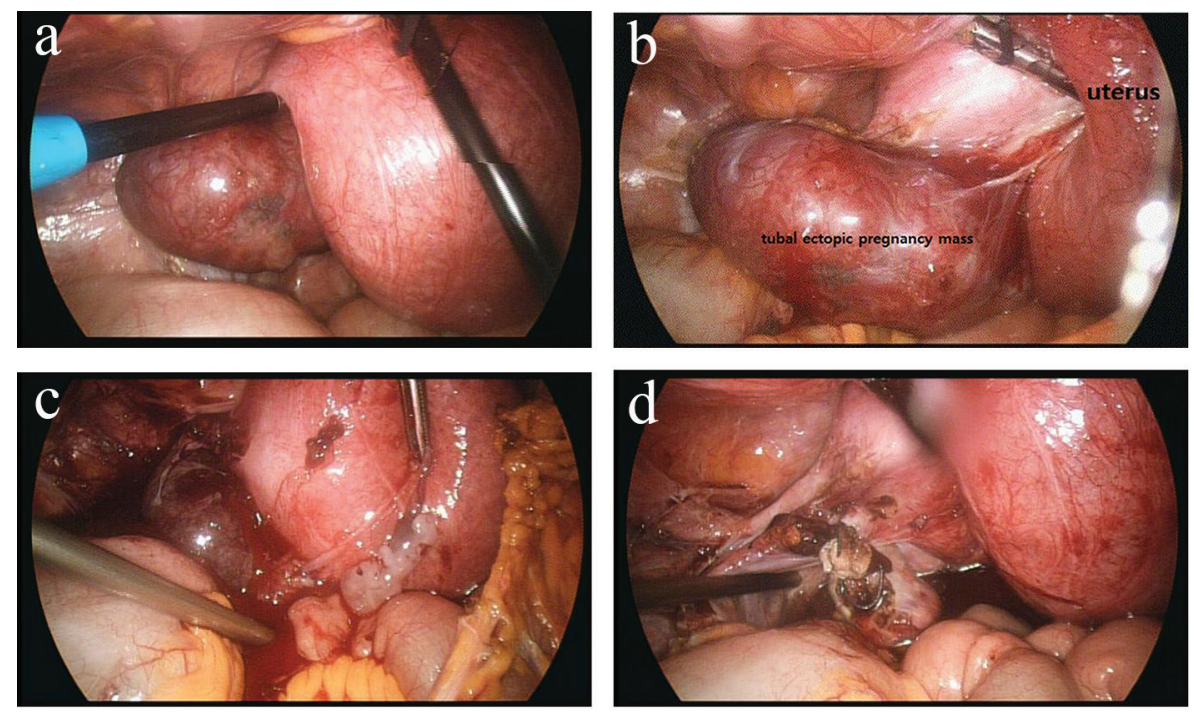

Figure 2. Laparoscopic view: dark bluish-colored unruptured tubal mass upon entering pelvic cavity (a), tubal mass adherent to uterus (b), ruptured mass during dissection and embryo expelled into abdominal cavity (c) and after laparoscopic left salpingectomy (d). 
cy mass size is usually $1.5-3.5 \mathrm{~cm}[2,3]$. The Fallopian tube, which has no elastic myometrium, ruptures if a tubal ectopic mass grows beyond a certain size. In this case, an ectopic mass measuring $7.3 \times 4.0 \mathrm{~cm}$ was detected on TVUS in the left adnexa in a woman with bilateral tubal obstruction on hysterosalpingography. An embryo with CRL consistent with 8 weeks, 4 days was noted in the ectopic mass, and was unusually large. To the author's knowledge, this is the largest reported, unruptured, singleton tubal ectopic pregnancy mass. There are some case reports of large tubal ectopic masses. Greene reported a singleton ectopic tubal pregnancy mass measuring up to $20 \mathrm{~cm}$ [11]. This is the largest reported. However, strictly speaking, this case was an abdominal pregnancy from a missed abortion in a tubal pregnancy. At laparotomy, the left tube was intact. A twin tubal pregnancy mass tends to show marked distension of the Fallopian tube without tubal rupture $[12,13]$. Krzaniak reported a twin tubal ectopic pregnancy mass measuring $10 \mathrm{~cm}$ [12]. Laparotomy with right salpingectomy was performed. Macroscopic examination of the removed tube revealed a twin pregnancy corresponding to about 12 weeks of gestation. Goswami et al reported a twin tubal ectopic pregnancy mass measuring $6 \mathrm{~cm}$ [13]. Laparotomy with right salpingectomy was carried out. The tube contained two embryos in separate gestational sacs with CRL of $2 \mathrm{~cm}$, corresponding to 8 weeks, 4 days of gestation. This suggests that tubal distension may not be the main factor causing tubal rupture in ectopic pregnancy. Although a twin pregnancy may be larger, trophoblastic invasion may be less, due to an earlier gestation, compared with an equivalent-sized singleton pregnancy, and is therefore less likely to rupture [13]. Although the present case was a singleton tubal ectopic pregnancy, it grew to $7.3 \mathrm{~cm}$ without rupture. It is assumed that a blastocyst was implanted in a fused Fallopian tube that was adherent to the pelvic peritoneum and uterus, and that the adherent surrounding structures prevented rupture. The present case was managed laparoscopically, unlike the previous cases. Laparoscopic left salpingectomy and adhesiolysis was performed. The guiding principle has become medical treatment, rather than surgery. Surgery is preferred in the case of a large ectopic pregnancy mass, and an embryo with an active fetal heartbeat. Even when both Fallopian tubes are obstructed, pregnancy is still possible. Laboratory studies should always include a pregnancy test when a patient complains of vaginal bleeding and abdominal pain.

\section{Conclusion}

This ectopic tubal pregnancy was unusual because of the large size in a distended and unruptured tube. To the author's knowledge, this is the largest reported, unruptured, singleton tubal ectopic pregnancy, measuring up to $7.3 \mathrm{~cm}$ without a hematoma. It is assumed that a blastocyst was implanted in a fused Fallopian tube that was adherent to the pelvic peritoneum and uterus, and that the adherent surrounding structures prevented rupture.

\section{Conflicts of Interest}

The author declares no conflicts of interest regarding the publication of this paper.

\section{References}

1. Lau S, Tulandi T. Conservative medical and surgical management of interstitial ectopic pregnancy. Fertil Steril. $1999 ; 72(2): 207-215$.

2. Gamzu R, Almog B, Levin Y, Pauzner D, Lessing JB, Jaffa A, Bar-Am A. The ultrasonographic appearance of tubal pregnancy in patients treated with methotrexate. Hum Reprod. 2002;17(10):2585-2587.

3. Balci O, Ozdemir S, Mahmoud AS, Acar A, Colakoglu MC. The efficacy of multiple-dose methotrexate treatment for unruptured tubal ectopic pregnancy and conversion rate to surgery: a study on 294 cases. Fertil Steril. 2010;93(7):2415-2417.

4. Murray H, Baakdah H, Bardell T, Tulandi T. Diagnosis and treatment of ectopic pregnancy. CMAJ. 2005;173(8):905912.

5. Ectopic pregnancy - United States, 1990-1992. MMWR Morb Mortal Wkly Rep. 1995;44(3):46-48.

6. Kamwendo F, Forslin L, Bodin L, Danielsson D. Epidemiology of ectopic pregnancy during a 28 year period and the role of pelvic inflammatory disease. Sex Transm Infect. 2000;76(1):28-32.

7. Creanga AA, Shapiro-Mendoza CK, Bish CL, Zane S, Berg CJ, Callaghan WM. Trends in ectopic pregnancy mortality in the United States: 1980-2007. Obstet Gynecol. 2011;117(4):837-843.

8. Barnhart K, Mennuti MT, Benjamin I, Jacobson S, Goodman D, Coutifaris C. Prompt diagnosis of ectopic pregnancy in an emergency department setting. Obstet Gynecol. 1994;84(6):1010-1015.

9. Connolly A, Ryan DH, Stuebe AM, Wolfe HM. Reevaluation of discriminatory and threshold levels for serum beta-hCG in early pregnancy. Obstet Gynecol. 2013;121(1):65-70.

10. Doubilet PM, Benson CB. Further evidence against the reliability of the human chorionic gonadotropin discriminatory level. J Ultrasound Med. 2011;30(12):1637-1642.

11. Greene R. An unusually large tubal pregnancy. West J Surg Obstet Gynecol. 1946;54:247-249.

12. Krzaniak S. Unilateral tubal twin pregnancy. Postgrad Med J. 1967;43(496):120-122.

13. Goswami D, Agrawal N, Arora V. Twin tubal pregnancy: A large unruptured ectopic pregnancy. J Obstet Gynaecol Res. 2015;41(11):1820-1822. 